\title{
Visual Attention Processes and Oculomotor Control in Autism Spectrum Disorder: A Brief Review and Future Directions
}

\author{
Stéphanie Bellocchi \\ University Paul-Valéry, Montpellier, France \\ Vincent Henry \\ Department of Child and Adolescent Psychiatry, \\ Autism Resources Center, CHRU Montpellier, France \\ Amaria Baghdadli \\ University Paul-Valéry, Montpellier, France \\ Department of Child and Adolescent Psychiatry, \\ Autism Resources Center, CHRU Montpellier, France
}

\begin{abstract}
Autism spectrum disorder (ASD) is defined as persistent deficits in social communication and social interaction, and restricted, repetitive patterns of behavior, interests or activities Diagnostic and Statistical Manual of Mental Disorders (5th ed., DSM-5; American Psychiatric Association, 2013). However, individuals with ASD show clearly atypical visual patterns. So far, indications of abnormal visual attention and oculomotor control concerning stimuli independent of social function in ASD have been found. The same findings have been shown in individuals suffering of other neurodevelopmental disorders (e.g., developmental coordination disorder and developmental dyslexia [DD]). Furthermore, visual attention processes and oculomotor control are supposed to be subserved by the magnocellular visual system, which has been found, in turn, to be dysfunctional in ASD and other neurodevelopmental disabilities (i.e., DD). The purpose of this article is to briefly review the link between oculomotor control and visual attention processes and ASD, and to discuss the specificity and overlap of eye movement findings between ASD and other neurodevelopmental disorders.
\end{abstract}

Keywords: autism spectrum disorder; visual attention processes; oculomotor control; comorbidity 
A utism spectrum disorder (ASD) is considered a neurodevelopmental disorder, and it is defined as persistent deficits in social communication and social interaction, and restricted, repetitive patterns of behavior, interests, or activities (Diagnostic and Statistical Manual of Mental Disorders, 5th ed. [DSM-5]; American Psychiatric Association [APA], 2013). This disorder is also characterized by an atypical pattern in joint attention and mutual eye gaze (Nation \& Penny, 2008) and reduced visual attention for social stimuli, such as human faces (e.g., Dawson et al., 2002). That is, an important amount of studies using face recognition tests show that individuals with ASD have specific impairment in recognizing faces (for a review, see Wang, Liu, \& Liu, 2015). For example, autistic children are worse in judging whether they had seen the faces before if compared to typically developing (TD) children. Electrophysiological studies confirm this pattern of results, highlighting that face processing impairments are present very early in autism, by 3 years of age (e.g., Dawson, Webb, \& McPartland, 2005). Moreover, as highlighted by Guillon, Hadjikhani, Baduel, and Rogé (2014), during the last decades, there has been an explosion of eye tracking studies investigating visual attention for social stimuli in ASD. These authors reviewed studies exploring social orienting, visually scanning of faces, and gaze following. Globally, most of these studies indicate that individuals with ASD have decreased visual attention to social stimuli relative to typical developing individuals. However, this atypical pattern appears not to be generalizable across context and to be more complex in nature. Guillon and colleagues suggested that basic social orienting ability in ASD is not qualitatively impaired in ASD but seems to be less efficient (Johnson, 2014).

One of the classical hypotheses used to explain the face processing impairment is that it derives from a pervasive problem in social interaction, and the reward and motivational value of social stimuli (e.g., Dawson et al., 2002; Dawson et al., 2005; Schultz, 2005; but see Ewing, Pellicano, \& Rhodes, 2013, for alternative hypothesis). One of the explanations as regards the nature of the origin of the social deficit, is that it might arise from a general impairment in empathizing and theory of mind (ToM; Baron-Cohen \& Belmonte, 2005), and from its precursors, that is, deficits in joint attention and imitation.

However, an alternative view is to try to see it differently, supporting the idea that the face processing impairment may not be entirely social in origin, but that a visual perceptual impairment independent of social function also contributes to this difficulty (Behrmann, Thomas, \& Humphreys, 2006). Moreover, according to the neuroconstructivist approach (see Karmiloff-Smith, 1998, for the original review), low-level attentional and perception atypicalities could cause impairments in the higher level cognitive modules. For example, Elsabbagh and colleagues (2011) showed that 9-month-old infants at familial risk for a later diagnosis of ASD differed from controls both in measurements of social perception and inhibitory control. They presented preliminary data from an ongoing longitudinal research program, suggesting clear associations between some of these measurements taken during infancy and autism-related characteristics at 3 years. In this perspective, the typical social and communicative "core" deficit in ASD could be the result of complex developmental interactions between attentional and social brain networks (e.g., Elsabbagh et al., 2011; Elsabbagh et al., 2013; Gliga, Bedford, Charman, \& Johnson, 2015; Jones \& Klin, 2013).

That is, a growing body of research is interested in visual perceptual skills concerning nonsocial stimuli in ASD patients. So far, indications of atypicalities in visual attention and oculomotor control in individuals with autism have been found in several studies (e.g., van der Geest, Kemner, Camfferman, Verbaten, \& van Engeland, 2001; for a comprehensive review, 
see also Rommelse, Van der Stigchel, \& Sergeant, 2008). The principal aim of this article is to briefly review the link between visual attention processes and oculomotor control concerning stimuli independent of social function, and ASD, and to discuss the specificity and overlap of eye movement findings between ASD and other neurodevelopmental disorders. In particular, even if it is possible and very interesting to discuss the link with other neurodevelopmental disorders - for example, ADHD—we will take here the cases of developmental dyslexia (DD) and developmental coordination disorder (DCD). To do that, we will employ a comprehensive approach that incorporates almost all of the accepted methods required to test the relationship between visual attention, oculomotor control, and higher order cognitive deficits. In particular, (a) we will discuss studies taking a prospective, longitudinal approach and their predictability with future neurodevelopmental disorders; (b) we will present comparison studies between participants with neurodevelopmental disorders and their controls; and (c) we will discuss some studies comparing impaired children with isolated disorders to those with both disorders.

\section{VISUAL PERCEPTUAL SKILLS IN INDIVIDUALS WITH AUTISM SPECTRUM DISORDER: WHAT DO WE REFER TO?}

First of all, it is important to note that different type of atypical visual-perceptual processes in ASD have been reported in the literature.

For example, individuals with ASD show superior visuospatial skills compared to TD ones. This occurs, for example, in the Embedded Figures Test (EFT; Witkin, Oltman, Raskin, \& Karp, 1971), which requires locating a shape hidden within a larger meaningful figure (Jolliffe \& Baron-Cohen, 1997; Shah \& Frith, 1993).

Supposedly, individuals with ASD are unrestrained by the Gestalt-inducing nature of the complex figure, and are able to quickly disassemble the figure to locate the hidden shape (Pellicano, Gibson, Maybery, Durkin, \& Badcock, 2005). In the same vein, individuals with ASD show better visuospatial abilities regarding the block design task attributed to a superior facility for segmentation of the design (Shah \& Frith, 1993). Furthermore, they have a local advantage on the Navon task (Plaisted, Swettenham, \& Rees, 1999). Traditionally, this last task consists of a global letter formed by the configuration of local letters (e.g., a global $S$ made of small $H \mathrm{~s}$ ). The global and the local letters were either consistent (e.g., global $S$, local $S$ s) or inconsistent (e.g., global $H$, local $S \mathrm{~s}$ ), and participants are instructed to respond to either the global letter or the local letter in a block of trials.

At a first level of analysis, these results have been explained with the hypothesis of weak central coherence establishing an enhanced processing of details in individuals with ASD (e.g., Happé, 1999). However, this advantage is associated with a difficulty: ASD individuals often, although not always, perform poorly in tasks where grouping is required (Brosnan, Scott, Fox \& Pye, 2004). Actually, conflicting results showed that when individuals with ASD are instructed to report the global target in the Navon task, they do not fail to do that (Plaisted et al., 1999). That is, these authors confirmed that weak central coherence in ASD is not because of an inability to integrate parts into wholes, but instead, might be a result of enhanced perception of local stimulus attributes.

At a second level of analysis, research has focused on the neural basis that can be responsible for the weak central coherence in ASD. In this sense, some studies suggest that individuals with ASD might show a deficit in the dorsal visual pathway (or magnocellular pathway; 
e.g., Milne et al., 2002). In the human visual system, the retina transmits visual input to the lateral geniculate nucleus (LGN) and then to the primary visual cortex via two main separate pathways: the magnocellular and parvocellular streams (Hendry \& Reid, 2000). In the extrastriate cortical regions the magnocellular cells provide the principal input to the dorsal stream leading to the dorsolateral occipital cortex and posterior parietal lobe regions. The magnocellular pathway (or magnocellular-dorsal [M-D] stream) is assumed to be involved in low-spatial-frequency processing and eye movement control, and it responds to rapidly changing stimuli, such as flicker and motion (Livingstone \& Hubel, 1987). In this sense, given that more rapid processing of the global level of a stimulus would require faster transmission of low spatial frequency information (Badcock, Whitworth, Badcock, \& Lovegrove, 1990), Milne and colleagues (2002) argued that deficit in the M-D stream might offer a physiological explanation of weak central coherence in ASD. Supporting the idea of a deficit in the M-D pathways in ASD, many studies showed that children with autism have difficulty detecting global, coherent motion (e.g., Milne et al., 2002; Ronconi et al., 2012). This constitutes another type of visual-perceptual process, where it is possible to observe atypicalities in the ASD population, a visual-perceptual process specifically linked to M-D pathways functioning as well as frontoparietal attentional integration processing.

In the coherent dot motion (CDM) task, children are required to determine the overall direction of coherently moving dots, set among a background of randomly moving dots. For example, some studies, found that high-functioning children with autism required a significantly higher percentage of dots to be moving coherently to be able to perceive global motion, compared with TD children of similar age and nonverbal abilities (e.g., Milne et al., 2002; Spencer et al., 2000). Taking into account the neural basis of this impairment, by means of a functional magnetic resonance imaging (fMRI) study, it has been recently shown that the reduced global motion perception in ASD is driven by an atypical early response in visual processing and may reflect a fundamental perturbation in neural circuitry (Robertson et al., 2014). However, it is important to say here that the M-D deficit explanation of perceptual atypicalities in the ASD group is far from being established and this hypothesis is currently under investigation.

Furthermore, Ronconi and collaborators (2012) recently aimed to explore the role of spatial attention in a coherent motion task by displaying coherently moving dots displayed in the central or peripheral visual field in children with ASD and TD children. They showed that in children with ASD, CDM perception was selectively impaired in the central condition. In addition, in the ASD group, CDM efficiency was correlated to the ability to zoom out the attentional focus, and autism symptoms severity was related to both the CDM and attentional zooming-out impairment. Again, Ronconi, Gori, Ruffino, Molteni, and Facoetti (2013) explored the hypothesis of an attentional zooming-out impairment more in depth in children with ASD, and they confirm the results previously presented. They conclude that this atypical attentional focusing, which seems to be specific in children with ASD, is probably linked to a dysfunctional top-down feedback from frontoparietal network to the early visual areas, and it could contribute to the atypical visual perception associated with these individuals. In turn, they conclude that these attentional impairments could have an impact on the social communicative development of the children with ASD.

Recent longitudinal studies showed that infants who develop autism show atypicality in the development of visual attention skills from the first year of life. In particular, regarding visual orienting, Elsabbagh and colleagues (2013) showed that at 7 months of age, disengagement was not robustly associated with later diagnostic outcomes of ASD. However, by 
14 months, they observed longer latencies to disengage in the risk group later diagnosed with autism than other infants at risk and the low-risk control group. Again, patterns of eventrelated potential components evoked in response to dynamic eye gaze shifts during infancy were associated with ASD diagnosed at 36 months (Elsabbagh et al., 2012). In the same vain, enhanced visual search performance on letter targets at 9 months predicted a higher level of autism symptoms at 15 months and at 2 years (Gliga et al., 2015). These results suggested that infant perceptual atypicalities are linked to the emerging autism phenotype, even if perceptual and social interaction patterns could reflect biologically independent pathologies (Happé \& Ronald, 2008). Finally, attentional abnormalities that are commonly seen in infants at biological risk (because of an older siblings affected) can be found also in infants whose parents have no ASD diagnosis but high autistic (Ronconi et al., 2014).

Furthermore, the enhanced visual processing of detailed information preference in individuals with ASD has been also highlighted with the visual crowding effect. This effect refers to the negative effect occurring when visual distractors are added around a central target which has to be identified (e.g., Bouma, 1970). As Huckauf and Heller (2004) mentioned, the processes on which the crowding effect is based are still unknown, and the same effect has been characterized with many different terms and specified by many different features. Traditionally, the reduced discriminability of the target surrounded by other similar visual stimuli is thought to be attributed to a "lateral masking," suggesting that crowding is caused by inhibitory neural interactions at an early level of visual processing (see Bellocchi, 2013, for a review on the explanations). Thus, Baldassi and collaborators (2009) showed that the crowding effect of eight flankers surrounding a small peripheral target is virtually absent in the group of children with high-functioning autism, indicating a superior ability to segregate cluttered visual items. In other words, these children are capable of segregating small stimuli in the presence of crowding flankers known to impair identification in TD individuals. However, this study failed to show a visual search superiority of the ASD group in a central presented configuration, as proposed in the previous cited studies.

More interestingly, Ronconi, Gori, Giora, and colleagues (2013) wanted to explore the spatial-temporal dynamics of visual processing in ASD by using attentional masking (AM), which consists in a reduction in the visibility of a target followed by a second object, which acts as a competitive stimulus (Ruffino et al., 2010). The authors highlighted that in the AM paradigm the competitive object appears on the visual scene with a specific temporal delay from the target object. This aspect differentiated this task from other static visual tasks (e.g., visual search and crowding), in which this temporal aspect is not involved. They thus compared the performances of the ASD group with that of TD children. They found that ASD and TD children did not differ in the AM effect provoked by the competitive object displayed in the same position of the target. In contrast, children with ASD showed a deeper and prolonged interference than the TD group when the masking object was displayed in the lateral position. In other words, they exhibit an efficient ability to allocate temporal attention onto the target, inhibiting the process of subsequent irrelevant information displayed in the same spatial location, but they fail to allocate attention to a lateral object with a different spatial-temporal position compared to the central target. These findings suggested that individuals with ASD suffer from a higher interference of lateral competitive objects than TD controls, despite their narrow focused attention (e.g., Ronconi et al., 2012). According to the "reentrant theory of perception” (Di Lollo, Enns, \& Rensink, 2000), Ronconi, Gori, Giora, and colleagues (2013) suggested that the irrelevant lateral object cannot be efficiently ignored by the individuals 
with ASD, because they suffer of a stronger masking object perceptual representation in the lower level of visual hierarchy (consequence of an abnormal lateral connectivity making the mask more salient) coupled with weak feedback projections from higher level responsible for a weak attentional selection at the locus of the target object.

The importance of the temporal dynamics of visual processing, which could be a crucial element for the altered visual processing in individuals with ASD, has been also previously considered in the rapid visual-motion integration deficit hypothesis (Gepner, 2001; Gepner \& Mestre, 2002). The idea here is that the movements in everyday life are too fast, and for this reason, individuals with ASD do not perceive them or they fail to do so. As a consequence, these defects of visual perception and visual-motor integration of movement (physical) could not only be responsible for early abnormalities in the development of motor anticipation, visual-postural adjustment and postural motor skills, but also have a negative impact of the awareness of continuity and body unity. Moreover, these authors argued that the deficit in the rapid visual-motor integration could explain why individuals with ASD have: severe difficulties in processing fast-moving visual events, higher motion-coherence thresholds than normal children, difficulties in motion direction discrimination, weak postural reactivity to visually perceived environmental motion, difficulties in facial recognition tasks involving the processing of facial dynamics (i.e., emotional expressions and movements of the lips and eyes, which are essentially rapid movements; Gepner \& Mestre, 2002).

A significant number of current studies agree on the link between attention and oculomotor control (but see Mazer, 2011). The regions involved in the control of eye movements, such as the frontal eye fields, the lateral intraparietal area, and the superior colliculus, are strictly involved in covert visual attention (e.g., Doré-Mazars, Pouget, \& Beauvillian, 2004). In this vein, eye movements constitute an excellent means of investigating visuospatial attention (Bellocchi, Muneaux, Bastien-Toniazzo, \& Ducrot, 2013). This issue will be briefly presented in the next section of this article.

\section{OCULOMOTOR CONTROL IN AUTISM SPECTRUM DISORDER}

Recording eye movements provides various benefits over standard procedures. First of all, eye movements provide information on online cognitive processes involved in a task. In this context, eye movements offer important additional information (i.e., not only about the process measured in the task) regarding the metrics and dynamics of oculomotor control, such as saccade size, trajectories of saccades, number of fixations, gaze duration, and refixation. However, we can also obtain information about characteristics of low-level visual-motor control, referring more specifically to the neurophysiological mechanisms involved in oculomotor control, such as binocular coordination, saccades, vergence, and combined movements. This constitutes another approach to the study of eye movements compared to the first one. One of the aims of this brief review is to make a distinction between studies developed within the first and the second approach. Finally, and more interestingly for the aim of this article, eye movements recording is a noninvasive technique, and it uses paradigms which are very simple and not difficult to perform by children and often require no advanced cognitive skills such as language, reading, or complex motor responding.

Furthermore, in general, measurement of eye movements has been employed so far to study the neuropathology of childhood and adolescent psychiatric disorders. In particular, visually guided saccades, antisaccades (ASs), memory-guided saccades (MGSs), and smooth 
pursuit have been measured not only in ASD but also in attention deficit hyperactivity disorder (ADHD), oppositional deviant disorder, conduct disorder, childhood-onset schizophrenia, Tourette's syndrome, obsessive-compulsive disorder, anxiety, and depression (for comprehensive reviews, see Karatekin, 2007, and Rommelse et al., 2008). Specifically, studies exploring eye movements in individual with ASD have focused in a very broad range of domains that are very different from each other. It is not the objective to give a comprehensive description of all the domains here, but we will focus more on studies according to the two approaches in the eye movement measurements described earlier.

Given that the major impairments of individuals with ASD lie in social skills and communication, most eye movement studies taking into account this neurodevelopmental disorder have focused (and are still focusing nowadays) on scan patterns of social scenes and facial expressions. For example, Dalton, Nacewicz, Alexander, and Davidson (2007) showed that children with ASD were less accurate and, consequently, their fixation durations were shorter compared to TD children in a face recognition task (Dalton et al., 2007; Dalton et al., 2005). Again, participants with high-functioning ASD showed less proportion of time on eyes and higher proportion of time on mouth, bodies, and objects compared to controls in a face and scene perception task (Klin, Jones, Schultz, Volkmar, \& Cohen, 2002). However, results in these types of studies are really inconsistent. Some authors found impairments in emotion recognition and time spent on eye region such as the eyes, and others did not (e.g., de Jong, van Engeland, \& Kemner, 2008). In that article, de Jong and colleagues (2008) found that ASD subjects performed normally when neutral faces were used. However, emotional faces elicited modified face and gaze cue processing in control subjects, but not in the ASD subjects.

However, according to the hypothesis stated in the paragraph earlier, face processing impairment could not be entirely social in origin, but a visual perceptual (which could be attentional in nature) impairment may also contribute to this difficulty (e.g., Behrmann et al., 2006). In this sense, it is necessary here to summarize the main findings, focusing on the assessment of basic oculomotor behavior and frontal circuitry in children with autism, which refer to visual attention processes and more low-level visual-motor control. We will notice that these studies are quite few in number.

In their review, Rommelse and colleagues (2008) reported studies focusing on paradigms providing information on cognitive processes functioning, such as top-down inhibition and memory (respectively with ASs — where participants are required to make an eye movement away from the onset location to its mirror position-and MGS - the participant is required to make a saccade toward the memorized location, only when he or she is allowed), and paradigms providing information on low-level visual-motor control, such as smooth pursuit eye movements (SPEMs) and visually guided saccades (VGSs). In the SPEM, the participant has to track a small object in motion with a constant (slow) speed, whereas in the VGS, participants are instructed to make an eye movement to a visual stimulus presented in the periphery. However, the authors claimed that very few studies have been conducted in these domains of investigation with individuals suffering of ASD.

It is important to specify here that, as stated by Scerif and colleagues (2005), the ability to inhibit saccades toward suddenly appearing peripheral stimuli (prosaccades) and direct them to contralateral locations instead (ASs), is a crucial marker of eye movement control. TD infants as young as 4 months old can learn to inhibit reflexive saccades to peripheral stimuli, but they do not produce ASs, for which the development occurs later in infancy, and their underlying neural computations remain, however, unexplored. 
As regards inhibitory control assessed with AS task, some studies showed more directional errors in autistic participants compared to controls; however results seem to be nonconsistent regarding the time needed to execute a correct AS. Furthermore, some studies suggest that the basic deficit in response inhibition seems to be present throughout development. In particular, a very recent fMRI study using an AS task, suggested that brain circuitry underlying inhibitory control develops differently from adolescence to adulthood in ASD. Specifically, there may be relative underdevelopment of brain processes underlying inhibitory control in ASD, which may lead to engagement of subcortical compensatory processes (Padmanabhan et al., 2015). Regarding VGS, it seems that no deficit emerges for individuals with ASD; however, once again, results seem to be inconsistent. For this last reason, no conclusions could be drawn on attentional engagement/disengagement in individuals with ASD assessed with the VGS paradigm. Regarding SPEM, however, some studies claimed the presence of an atypical behavior, suggesting that various noncortical areas may also be involved in the neuropathology of ASD (Rommelse et al., 2008). This result has been recently replied by Wilkes, Carson, Patel, Lewis, and White (2015), showing that children with high-functioning ASD exhibited greater horizontal saccade latency and greater phase lag during vertical smooth pursuit.

However, using an anticipatory smooth eye movements' paradigm (defined as smooth eye movements in the direction of expected future motion), adults with high-functioning ASD showed strong anticipatory smooth eye movements that had a velocity the same as that of a group of neurotypical participants. These results, contrary to the ones shown by traditional SPEM, showed that anticipatory capacities are intact in high-functioning ASD in cases where the cue to the motion path is highly salient and unambiguous (Aitkin, Santos, \& Kowler, 2013). It is thus suggested that the ability to make predictions and engage in at least one type of predictive motor behavior is present in high-functioning ASD, a cognitive skill that is fundamental for the interpretation of events in natural environments or social situations.

So far, we can easily realize that the integrity of the oculomotor system within ASD population is unclear, with inconsistent results of atypicalities in eye movements in basic oculomotor control tasks.

More interestingly, a recent study aimed at clarifying if these contrasting results could be an expression of the heterogeneity of this clinical population (Kelly, Walker, \& Norbury, 2013). To do that, these researchers explored reflexive (i.e., prosaccades) and volitional eye movement control (i.e., AS task) in 8- to 14-year-old children who are autism language normal (ALN), autism language impaired (ALI), nonautistic language impaired (LI), and TD. As other studies showed (see Rommelse et al., 2008), there were no differences regarding reflexive eye movements between groups, suggesting that at the most basic level, the underlying oculomotor system is intact. However, deficits in AS task were found to be linked with language status, and were not specific to ASD. In particular, the authors reported that more than $80 \%$ of ALI and LI children had error rates at least 1.5 standard deviations (SDs) below the TD mean in an antisaccade task. Moreover, in the search distracter task, $35.29 \%$ of ALI children and $43.75 \%$ of LI children had error rates greater than 1.5 SDs compared with $17.64 \%$ of ALN children. These results were similar to those previously found by Takarae, Minshew, Luna, and Sweeney (2004) who reported that oculomotor control deficits in ASD, identified using a VGS task, were associated with language delay. Moreover, reinforcing the link between attentional engagement deficit and language impairments, it has been found that children with SLI showed a sluggish engagement of temporal attention (Dispaldro et al., 
2013). Taken as a whole, these results suggested that there are phenotypic overlaps between ALI and LI populations in oculomotor control (i.e., AS task), throughout the role of language mediation. This could mean that volitional control of eye movements may serve as a marker of neurodevelopment anomaly, in which language acquisition is especially vulnerable.

Thus, the intriguing question of the specificity and overlap of visual attention processes and oculomotor control between ASD and other neurodevelopmental disorders will be discussed in the next final section taking into account, in particular, the cases of DD and DCD.

\section{DISCUSSION: SOME ISSUES ON SPECIFICITY AND OVERLAPS BETWEEN AUTISM SPECTRUM DISORDER AND OTHER NEURODEVELOPMENTAL DISORDERS IN VISUAL ATTENTION PROCESSES AND OCULOMOTOR CONTROL}

As stated before, ASD is defined as persistent deficits in social communication and social interaction and restricted, repetitive patterns of behavior, interests or activities (DSM-5; APA, 2013). Despite these common core features, ASD is characterized by huge heterogeneity in symptom severity and associated impairment in other domains of development, such as cognitive ability, language skills, and adaptive behavior. As we have anticipated before by presenting the study of Kelly and colleagues (2013), ASD could frequently be associated with language impairments, which can produce different profiles and different developmental trajectories of the ASD itself. The latest edition of DSM-5 (APA, 2013) represents an attempt to organize this heterogeneity and comorbidity between different disorders by using a dimensional approach. Thus, according to this new classification, ASD is included in a broader category that refers to "neurodevelopmental disorders." This category also includes other neurodevelopmental disabilities such as $D D$ and $D C D$, among others. In particular, $D D$ is diagnosed when no sensory and intellectual deficits can explain reading and/or writing disorders and when adequate instruction and sociocultural opportunities are available but fail to result in an adequate level of performance. Furthermore, the $D C D$ concerns difficulties with various aspects of the motor skills, from walking, the global coordinations, the balance, up to the fine abilities, such as drawing or writing. In this final section, we will thus discuss some similarities and some differences between ASD, DD, and DCD regarding the findings presented in the two previous sections about visual attention processes and oculomotor control.

Sensory theories of DD suggest that the basis of this disorder could be a deficit that is sensory in nature (e.g., Goswami, 2014; Stein \& Walsh, 1997). Indeed, in the past decades, a very large amount of studies have shown that, despite phonological deficits, several dyslexics have a deficit of visual processing (e.g., Boden \& Giaschi, 2007, for a review; Spinelli, De Luca, Judica, \& Zoccolotti, 2002; Stein, 2001; Stein \& Walsh, 1997). As in individuals with ASD, dyslexics are less sensitive to global, coherent motion (e.g., Conlon, Lilleskaret, Wright, \& Power, 2012; Cornelissen, Richardson, Mason, Fowler, \& Stein, 1995; Pellicano \& Gibson, 2008; Talcott et al., 2003). Again, at the biological level, these visual deficits are attributed to a dysfunction of the magnocellular pathway (or M-stream), which is assumed to be involved in low-spatial-frequency processing and eye movement control. In particular, Gori, Cecchini, Bigoni, Molteni, and Facoetti (2014) suggested that an M-D deficit might impair the sublexical mechanisms that are critical for reading development. Indeed, recent studies confirm that illusory visual motion perception — specifically processed by the M-D stream-is impaired in children with DD (e.g., Gori, Mascheretti, et al., 2015). Thus, it is possible that 
ASD and dyslexia share a common visual-motion integration impairment, at least at a visualoculomotor level. However, more precisely, Pellicano and Gibson (2008) showed that there are some differences. Indeed, although children with autism demonstrated intact lower level, but impaired higher level dorsal-stream functioning, children with dyslexia displayed abnormalities at both lower and higher levels of the dorsal visual stream. The authors claimed that these findings suggest that these disorders can be dissociated according to the origin of the impairment along the dorsal-stream pathway. Moreover, given the same phenotypic profile (e.g., decreased coherent motion performance; e.g., Conlon et al., 2012; Cornelissen et al., 1995; Pellicano \& Gibson, 2008; Talcott et al., 2003), the underlying deficit could be different in nature. Although for dyslexics, very recent longitudinal studies seem to support the idea that a dysfunction of the M-D pathway could be seen as one of the causal factors (e.g., Gori, Seitz, Ronconi, Franceschini, \& Facoetti, 2015; for a multiple deficit view of dyslexia, see, e.g., Pennington, 2006), the same cannot be said for ASD, where many other explanations could be found and where robust causal evidence have not been reported yet.

Furthermore, visual attention and oculomotor deficits have been observed in DD (e.g., see Bellocchi, Muneaux, et al., 2013, for a comprehensive review).

That is, on the one hand, some studies reported a "sluggish attentional shifting" (Hari \& Renvall, 2001; Facoetti, Lorusso, Paganoni, Umiltà, \& Mascetti, 2003), and attention deficits in the automatic orienting and focusing of spatial attention (Facoetti, Paganoni, Turatto, Marzola, \& Mascetti, 2000), as well an asymmetrical allocation of attention to the right visual field in dyslexia, which has been interpreted as a left mini-neglect phenomenon (Facoetti, Turatto, Lorusso, \& Mascetti, 2001) or a reduced-visual attention span (Valdois, Bosse, Tainturier, 2004). In addition, it is interesting to note that, regarding the AM presented in the previous section, children with dyslexia and specific language impairment, as those with ASD, show a stronger central AM effect in comparison to the control groups (Dispaldro et al., 2013; Lum, Conti-Ramsden, \& Lindell, 2007; Ruffino et al., 2010). However, regarding the crowding effect, there seems to be some differences between individuals with ASD and dyslexics, because the former do not seem to be sensitive to this effect (Baldassi et al., 2009), contrary to the second population. That is, it seems that, in several dyslexic children, abnormal crowding impeded the development of a faster reading procedure (e.g., Bellocchi, 2013; Gori \& Facoetti, 2015; Spinelli et al., 2002).

On the other hand, some studies showed that dyslexic readers had an abnormally longer latency for saccades and vergence, and poor binocular coordination of saccades and fixations when exploring paintings, suggesting an oculomotor deficit (Bucci, Brémond-Gignac, \& Kapoula, 2008; Kapoula et al., 2008). These studies explored low-level oculomotor control. However, others investigated oculomotor control, which is specific to reading or word recognition. For example, some studies focused on saccade computation, defined as the positioning where the eyes land on a word, (i.e., optimal viewing position [OPV] and preferred viewing location effects; e.g., Rayner, 1979) in dyslexic readers. Although very few studies have been conducted in this domain, Ducrot and colleagues (Lété, Sprenger-Charolles, Pynte, \& Billard, 2003; Ducrot, Pynte, Ghio, \& Lété, 2013) found that dyslexic children showed differences in their patterns of viewing position effects, and, even though they showed an OVP effect as normal readers do, dyslexics had a symmetrical curve. In particular, it has been showed that fifth grade typical reading children take the direction of attentional scanning (left-to-right) into account, which results in asymmetrical landing-position pattern. This mechanism depends on the discreteness of the stimulus and, interestingly, it is less irrepressible in lower reading level children 
and in children with reading disability. This difficulty does not seem to be a dysfunction, but rather a delay, that is, a mirror of their lower reading exposure (Bellocchi, Mancini, et al., 2013). On the contrary, when DD is associated with motor disorder (i.e., DCD), a dysfunction in saccade computation is observable (Bellocchi et al., 2015). Moreover, the initial fixation position of dyslexics in word recognition is not "optimal," and positioning errors are more frequent, leading to more refixations than normal readers (Hawelka, Gagl, \& Wimmer, 2010). Finally, these processes are specifically linked to reading, and they have not yet been explored in individuals with ASD. Even if it is known that individuals with ASD have, in general, intact decoding skills, some of them can show poor reading abilities (White et al., 2006). That is, the presence of atypicalities in landing position pattern in this subgroup of individuals with ASD can be considered for future studies.

Many studies have shown that dyslexia is frequently associated with DCD (for a comprehensive review, see Jover et al., 2013). In particular, some studies showed that dyslexic children have more difficulties compared to their peers to perform tests assessing visual-motor integration skills (e.g., pegboard test [Nicolson \& Fawcett, 1994], bead threading [Fawcett \& Nicolson, 1995], and pointing [Velay, Daffaure, Giraud, \& Habib, 2002]). Indeed, recently it has been found that dyslexics showed lower motor-reduced visual perception and visualmotor integration abilities compared to normal readers, both chronological age and reading level matched controls (Bellocchi et al., under review). This means that, regarding the visual perception and visual motor integration abilities, the dyslexic group is characterized by a specific deficit and not by a developmental delay (Goswami, 2003, 2014). More interestingly, compared to children with DCD, children with dyslexia showed higher motor-reduced visual perception and higher visual-motor integration. Those results suggest that motor-reduced visual perception can discriminate between isolated disorders, particularly in children with dyslexia only and children with DCD only. Furthermore, visual-motor integration can discriminate between children who have DCD and children who have not. Thus, this study highlights different profiles between children with DD and children with DCD. Furthermore, aiming at exploring the link between motor disabilities and ASD, some researchers administered a broad range of motor tests in this population. They found that controlling for IQ, gender, and age, the children with ASD performed significantly worse on ideational and buccofacial praxis, simple motor skill, timing and accuracy of saccadic eye movements, motor coordination, and visual-motor integration (Miller, Chukoskie, Zinni, Townsend, \& Trauner, 2014; for a review, see Fournier, Hass, Naik, Lodha, \& Cauraugh, 2010). These results show that ASD could be associated with motor disabilities, even if it seems that it is premature to designate motor disabilities as a core symptom of ASD. In particular, regarding oculomotor control and visual attention, this study explored performances of children in the gap/null/ overlap paradigm, which consists of examine the effect of early (gap condition), simultaneous (null condition), or late (overlap condition) removal of a central fixation on saccadic latency to a peripheral target stimulus. As we mentioned before, children with ASD showed deficit in executing this task because they were slower to initiate saccades and less accurate. The authors interpreted these results according to hypotheses implicating cerebellar modulation of movement plans (Fatemi et al., 2012; Ivry, 2003).

Finally, it has been observed that DCD was diagnosed in $25 \%$ of girls with ASD and ADHD (Kopp, Beckung, \& Gillberg, 2010).

To conclude, from a theoretical point of view, a multiple cognitive deficit model could be considered to explain the frequent association between specific neurodevelopmental 
disorders and sensorimotor manifestations, as we have stated in this brief review (Pennington, 2006). In this vein, according to Ramus (2004), motion perception deficit (or the underlying dorsal visual stream dysfunction) could be one of the factors involved in higher order cognitive deficits which are presented in ASD and in other neurodevelopmental disorders. Moreover, Mazer (2011) suggested that in typical developing individuals attentional capture by low-level cues (e.g., bright flash, fast movements) is effectively suppressed to enable tracking of the social interaction. On the contrary, individuals with ASD fail to suppressed attentional capture resulting in abnormalities in processing social stimuli. This implies that a deficit in the control system may be involved in some of the social impairments associated to ASD.

Second, according to the neuroconstructivist approach, the study of developmental disorders should be based on longitudinal studies from early childhood, in which several assessment sessions are implemented, to identify such problems as early as possible (Karmiloff-Smith, 1998; Goswami, 2003, 2014). This method allows researchers to investigate the causes of developmental disorders by considering the different developmental trends that can lead to different phenotypic outcomes. In particular, exploring visual attention precursors, as they are highlighted by oculomotor skills, by exploring TD children and children at familial risk for ASD, could be useful for early identification of children at risk for ASD.

\section{REFERENCES}

Aitkin, C. D., Santos, E. M., \& Kowler, E. (2013). Anticipatory smooth eye movements in autism spectrum disorder. Plos One, 8(12), e83230.

American Psychiatric Association. (2013). Diagnostic and statistical manual of mental disorders (5th ed.). Washington, DC: Author.

Badcock, J. C., Whitworth, F. A., Badcock, D. R., \& Lovegrove, W. J. (1990). Low-frequency filtering and the processing of local-global stimuli. Perception, 19, 617-629

Baldassi, S., Pei, F., Megna, N., Recupero, G., Viespoli, M., Igliozzi, R., . . Cioni, G. (2009). Search superiority in autism within, but not outside the crowding regime. Vision Research, 49, 2151-2156.

Baron-Cohen, S., \& Belmonte, M. K. (2005). Autism: A window onto the development of the social and the analytic brain. Annual Review Neuroscience, 28, 109-126.

Behrmann, M., Thomas, C., \& Humphreys, K. (2006). Seeing it differently: Visual processing in autism. Trends in Cognitive Sciences, 10(6), 258-263.

Bellocchi, S. (2013). Developmental dyslexia, visual crowding and eye movements. In L. C. Stewart (Ed.), Eye movement: Developmental perspectives, dysfunctions and disorders in humans (pp. 93-110). New York, NY: Nova Science Publishers.

Bellocchi, S., Huau, A., Jover, M., Brun-Hénin, F., Mancini, J., \& Ducrot, S. (2015). Oculomotor control in DCD children with and without developmental dyslexia: What is the impact of co-occurrence between neurodevelopmental disorders? Journal of Comorbidity, 5, 48.

Bellocchi, S., Mancini, J., Jover, M., Huau, A., Ghio, A., André, C., \& Ducrot, S. (2013, AugustSeptember). Dyslexic readers and saccade computation: Effects of reading exposure and visuo-perceptual constraints. Poster session presented at the 18th ESCoP Conference, Budapest, Hungary.

Bellocchi, S., Muneaux, M., Bastien-Toniazzo, M., \& Ducrot, S. (2013). I can read it in your yes: What eye movements tell us about visuo-attentional processes in developmental dyslexia. Research in Developmental Disabilities, 34, 452-460.

Bellocchi, S., Muneaux, M., Huau, A., Leveque, Y., Jover, M., \& Ducrot, S. (under review). Exploring the link between visual perception, visual-motor integration, and reading in normal developing and impaired children using DTVP-2. Dyslexia. 
Boden, C., \& Giaschi, D. (2007). M-stream deficits and reading-related visual processes in developmental dyslexia. Psychological Bulletin, 133(2), 346-366.

Bouma, H. (1970). Interaction effects in parafoveal letter recognition. Nature, 226(5241), 177-178.

Brosnan, M. J., Scott F. J., Fox, S., \& Pye, J. (2004). Gestalt processing in autism: Failure to process perceptual relationships and the implications for contextual understanding. Journal of Child Psychology and Psychiatry, 45, 459-469.

Bucci, M. P., Brémond-Gignac, D., \& Kapoula, Z. (2008). Latency of saccades and vergence eye movements in dyslexic children. Experimental Brain Research, 188(1), 1-12.

Conlon, E. G., Lilleskaret, G., Wright, C. M., \& Power, G. F. (2012). The influence of contrast on coherent motion processing in dyslexia. Neuropsychologia, 50, 1672-1681.

Cornelissen, P., Richardson, A., Mason, A., Fowler, S., \& Stein, J. (1995). Contrast sensitivity and coherent motion detection measured at photopic luminance levels in dyslexics and controls. Vision Research, 35, 1483-1494. http://dx.doi.org/10.1016/0042-6989(95)98728-R

Dalton, K. M., Nacewicz, B. M., Alexander, A. L., \& Davidson, R. J. (2007). Gaze-fixation, brain activation, and amygdala volume in unaffected siblings of individuals with autism. Biological Psychiatry, $61,512-520$.

Dalton, K. M., Nacewicz, B. M., Johnstone, T., Schaefer, H. S., Gernsbacher, M. A., Goldsmith, H. H., ... Davidson, R. J. (2005). Gaze fixation and the neural circuitry of face processing in autism. Nature Neuroscience, 8, 519-526.

Dawson, G., Carver, L., Meltzoff, A., Panagiotides, H., McPartland, J., \& Webb, S. J. (2002). Neural correlates of face and object recognition in young children with autism spectrum disorder, developmental delay, and typical development. Child Development, 73, 700-717.

Dawson, G., Webb, S. J., \& McPartland, J. (2005). Understanding the nature of face processing impairment in autism: Insights from behavioral and electrophysiological studies. Developmental Neuropsychology, 27, 403-424.

de Jong, M. C., van Engeland, H., \& Kemner, C. (2008). Attentional effects of gaze shifts are influenced by emotion and spatial frequency, but not in autism. Journal of the American Academy of Child Q Adolescent Psychiatry, 47(4), 443-454.

Di Lollo, V., Enns, J. T., \& Rensink, R. A. (2000). Competition for consciousness among visual events: The psychophysics of reentrant visual processes. Journal of Experimental Psychology, Journal of Experimental Psychology. General, 129, 481-507.

Dispaldro, M., Leonard, L. B., Corradi, N., Ruffino, M., Bronte, T., \& Facoetti, A. (2013). Visual attentional engagement deficits in children with specific language impairment and their role in real-time language processing. Cortex, 49, 2126-2139.

Doré-Mazars, K., Pouget, P., \& Beauvillain, C. (2004). Attentional selection during preparation of eye movements. Psychological Research, 69, 67-76.

Ducrot, S., Lété, B., Sprenger-Charolles, L., Pynte, J., \& Billard, C. (2003). The viewing position effect in beginning and dyslexic readers. Current Psychology Letters: Behaviour, Brain \& Cognition, 10(1), 1-10. Retrieved from http://cpl.revues.org/document99.html

Ducrot, S., Pynte, J., Ghio, A., \& Lété, B. (2013). Visual and linguistic determinants of the eyes' initial fixation position in reading development. Acta Psychologica, 142, 287-298.

Elsabbagh, M., Fernandes, J., Jane Webb, J. S., Dawson, G., Charman, T., \& Johnson, M. H. (2013). Disengagement of visual attention in infancy is associated with emerging autism in toddlerhood. Biological Psychiatry, 74, 189-194.

Elsabbagh, M., Holmboe, K., Gliga, T., Mercure, E., Hudry, K., Charman, T., .. Johnson, M. H. (2011). Social and attention factors during infancy and the later emergence of autism characteristics. Progress in Brain Research, 189, 195-207.

Elsabbagh, M., Mercure, E., Hudry, K., Chandler, S., Pasco, G., Charman, T., ... Johnson, M. H. (2012). Infant neural sensitivity to dynamic eye gaze is associated with later emerging autism. Current Biology, 22, 338-342. 
Ewing, L., Pellicano, E., \& Rhodes, G. (2013). Using effort to measure reward value of faces in children with autism. PLoS One, 8(11), e79493.

Facoetti, A., Lorusso, M. L., Paganoni, P., Umiltà, C., \& Mascetti, G. G. (2003). The role of visuospatial attention in developmental dyslexia: Evidence from a rehabilitation study. Cognitive Brain Research, 15, 154-164.

Facoetti, A., Paganoni, P., Turatto, M., Marzola, V., \& Mascetti, G. (2000). Visuospatial attention in developmental dyslexia. Cortex, 36, 109-123.

Facoetti, A., Turatto, M., Lorusso, M. L., \& Mascetti, G. G. (2001). Orienting of visual attention in dyslexia: Evidence for asymmetric hemispheric control of attention. Experimental Brain Research, 138, 46-53.

Fatemi, S. H., Aldinger, K. A., Ashwood, P., Bauman, M. L., Blaha, C. D., Blatt, G. J., . . Welsh, J. P. (2012). Consensus paper: Pathological role of the cerebellum in autism. Cerebellum, 11, 777-807.

Fawcett, A. J., \& Nicolson, R. I. (1995). Persistent deficits in motor skill of children with dyslexia. Journal of Motor Behavior, 27(3), 235-240.

Fournier, K. A., Hass, C. J., Naik, S. K., Lodha, N., \& Cauraugh, J. H. (2010). Motor coordination in autism spectrum disorders: A synthesis and metaanalysis. Journal of Autism and Developmental Disorders, 40, 1227-1240.

Gepner, B. (2001). "Malvoyance” du mouvement dans l'autisme infantile ? Une nouvelle approche neuropsychopathologique développementale ["Visually impaired" movement in infantile autism? A new developmental neuropsychopathological approach]. La Psychiatrie de l'Enfant, 1, 77-126.

Gepner, B., \& Mestre, D. (2002). Rapid visual-motion integration deficit in autism. Trends in Cognitive Sciences, 6(11), 455.

Gliga, T., Bedford, R., Charman, T., \& Johnson, M. (2015). Enhanced visual search in infancy predicts emerging autism symptoms. Current Biology, 25, 1727-1730.

Gori, S., Cecchini, P., Bigoni, A., Molteni, M., \& Facoetti, A. (2014). Magnocellular-dorsal pathway and sub-lexical route in developmental dyslexia. Frontiers in Human Neuroscience, 8, 460.

Gori, S., \& Facoetti, A. (2015). How the visual aspects can be crucial in reading acquisition? The intriguing case of crowding and developmental dyslexia. Journal of Vision, 15(1), 1-20.

Gori, S., Mascheretti, S., Giora, E., Ronconi, L., Ruffino, M., Quadrelli, E., . . Marino, C. (2015). The DCDC2 intron 2 deletion impairs illusory motion perception unveiling the selective role of magnocellular-dorsal stream in reading (dis)ability. Cerebral Cortex, 25(6), 1685-1695.

Gori, S., Seitz, A., Ronconi, L., Franceschini, S., \& Facoetti, A. (2015). Multiple causal links between magnocellular-dorsal pathway deficit and developmental dyslexia. Cerebral Cortex, 1-14.

Goswami, U. (2003). Why theories about developmental dyslexia require developmental designs. Trends in Cognitive Sciences, 7(12), 534-540.

Goswami, U. (2014). Sensory theories of developmental dyslexia: Three challenges for research. Nature Reviews Neuroscience, 16, 43-54. http://dx.doi.org/10.1038/nrn3836

Guillon, Q., Hadjikhani, N., Baduel, S., \& Rogé, B. (2014). Visual social attention in autism spectrum disorder: Insights from eye tracking studies. Neurosciences Q B Biobehavioral Reviews, 42, 279-297.

Happé, F. (1999). Autism: Cognitive deficit or cognitive style? Trends in Cognitive Sciences, 3(6), 216-222.

Happé, F., \& Ronald, A. (2008). The "fractionable autism triad": A review of evidence from behavioural, genetic, cognitive and neural research. Neuropsychology Review, 18, 287-304.

Hari, R., \& Renvall, H. (2001). Impaired processing of rapid stimulus sequences in dyslexia. Trends Cognitive Science, 5, 525-532.

Hawelka, S., Gagl, B., \& Wimmer, H. (2010). A dual-route perspective on eye movements of dyslexic readers. Cognition, 115, 367-379.

Hendry, S. H. C., \& Reid, R. C. (2000). The koniocellular pathway in primate vision. Annual Review of Neuroscience, 23, 127-153.

Huckauf, A., \& Heller, D. (2004). On the relations between crowding and visual masking. Perception and Psychophysics, 66(4), 584-595. 
Ivry, R. B. (2003). Cerebellar involvement in clumsiness and other developmental disorders. Neural Plasticity, 10, 141-153.

Johnson, M. H. (2014). Autism: Demise of the innate social orienting hypothesis. Current Biology, 24(1), R30-R31.

Jolliffe, T., \& Baron-Cohen, S. (1997). Are people with autism and Asperger syndrome faster than normal on the Embedded Figures Test? Journal of Child Psychology and Psychiatry and Allied Disciplines, $38,527-534$.

Jones, W., \& Klin, A. (2013). Attention to eyes is present but in decline in 2-6 month-olds later diagnosed with autism. Nature, 504(7480), 427-431.

Jover, M., Ducrot, S., Huau, A., Bellocchi, S., Brun-Hénin, F., \& Mancini, J. (2013). Les troubles moteurs chez les enfants dyslexiques: Revue de travaux et perspectives [Motor disorders in dyslexic children: A review and some perspectives]. Enfance, 4, 323-347.

Kapoula, Z., Bucci, M. P., Ganem, R., Poncet, S., Daunys, G., \& Brémond-Gignac, D. (2008). Free exploration of painting uncovers particular loose yoking of saccades in dyslexic readers. Dyslexia, 15(3), 243-259.

Karatekin, C. (2007). Eye tracking studies of normative and atypical development. Developmental Review, 27, 283-348.

Karmiloff-Smith, A. (1998). Development itself is the key to understanding developmental disorders. Trends in Cognitive Science, 2(10), 389-398.

Kelly, D. J., Walker, R., \& Norbury, C. F. (2013). Deficits in volitional oculomotor control align with language status in autism spectrum disorders. Developmental Science, 16(1), 56-66.

Klin, A., Jones, W., Schultz, R., Volkmar, F., \& Cohen, D. (2002). Visual fixation patterns during viewing of naturalistic social situations as predictors of social competence in individuals with autism. Archives of General Psychiatry, 59, 809-816.

Kopp, S., Beckung, E., \& Gillberg, C. (2010). Developmental coordination disorder and other motor control problems in girls with autism spectrum disorder and/or attention-deficit/hyperactivity disorder. Research in Developmental Disabilities, 31, 350-361.

Livingstone, M. S., \& Hubel, D. H. (1987). Psychophysical evidence for separate channels for the perception of form, color, movement, and depth. Journal of Neuroscience, 7(11), 3416-3468.

Lum, J. A. G., Conti-Ramsden, G., \& Lindell, A. K. (2007). The attentional blink reveals sluggish attentional shifting in adolescents with specific language impairment. Brain and Cognition, 63, 287-295.

Mazer, J. A. (2011). Spatial attention, feature-based attention and saccades: Three sides of one coin? Biological Psychiatry, 69(12), 1147-1152.

Miller, M., Chukoskie, L., Zinni, M., Townsend, J., \& Trauner, D. (2014). Dyspraxia, motor function and visual-motor integration in autism. Behavioural Brain Research, 269, 95-102.

Milne, E., Swettenham, J., Hansen, P., Campbell, R., Jeffries, H., \& Plaisted, K. (2002). High motion coherence thresholds in children with autism. Journal of Child Psychology and Psychiatry, 43, 255-263.

Nation, K., \& Penny, S. (2008). Sensitivity to eye gaze in autism: Is it normal? Is it automatic? Is it social? Development and Psychopathology, 20, 79-97.

Nicolson, R. I., \& Fawcett, A. J. (1994). Comparison of deficits in cognitive and motor skills among children with dyslexia. Annals of Dyslexia, 44, 147-164.

Padmanabhan, A., Garver, K., O’Hearn, K., Nawarawong, N., Liu, R., Minshew, N., . . Luna, B. (2015). Developmental changes in brain function underlying inhibitory control in autism spectrum disorders. Autism Research, 8(2), 123-135.

Pellicano, E., \& Gibson, L. Y. (2008). Investigating the functional integrity of the dorsal visual pathway in autism and dyslexia. Neuropsychologia, 46, 2593-2596.

Pellicano, E., Gibson, L. Y., Maybery, M., Durkin, K., \& Badcock, D. R. (2005). Abnormal global processing along the dorsal visual pathway in autism: A possible mechanism for weak central coherence. Neuropsychologia, 43, 1044-1053. 
Pennington, B. F. (2006). From single to multiple deficit models of developmental disorders. Cognition, 101, 385-413.

Plaisted, K., Swettenham, J., \& Rees, L. (1999). Children with autism show local precedence in a divided attention task and global precedence in a selective attention task. Journal of Child Psychology and Psychiatry and Allied Disciplines, 40(5), 733-742.

Ramus, F. (2004). Neurobiology of dyslexia: A reinterpretation of the data. Trends in Neuroscience, 27, $720-726$.

Rayner, K. (1979). Eye guidance in reading: Fixation location within words. Perception, 8, 21-30.

Robertson, C. E., Thomas, C., Kravitz, D. J., Wallace, G. L., Baron-Cohen, S., Martin, A., \& Baker, C. I. (2014). Global motion perception deficits in autism are reflected as early as primary visual cortex. Brain, 137, 2588-2599.

Rommelse, N. N. J., Van der Stigchel, S., \& Sergeant, J. A. (2008). A review on eye movement studies in childhood and adolescent psychiatry. Brain and Cognition, 68, 391-414.

Ronconi, L., Facoetti, A., Bulf, H., Franchin, L., Bettoni, R., \& Valenza, E. (2014). Paternal autistic traits are predictive of infants visual attention. Journal of Autism and Developmental Disorders, 44, 1556-1564

Ronconi, L., Gori, S., Giora, E., Ruffino, M., Molteni, M., \& Facoetti, A. (2013). Deeper attentional masking by lateral objects in children with autism. Brain Cognition, 82, 213-218.

Ronconi, L., Gori, S., Ruffino, M., Franceschini, S., Urbani, B., Molteni, M., \& Facoetti, A. (2012). Decreased coherent motion discrimination in autism spectrum disorder: The role of attentional zoomout deficit. PLoS One, 7, e49019.

Ronconi, L., Gori, S., Ruffino, M., Molteni, M., \& Facoetti, A. (2013). Zoom-out attentional impairment in children with autism spectrum disorder. Cortex, 49, 1025-1033.

Ruffino, M., Trussardi, A. N., Gori, S., Finzi, A., Giovagnoli, S., Menghini, D., Facoetti, A. (2010). Attentional engagement deficits in dyslexic children. Neuropsychologia, 48, 3793-3801.

Scerif, G., Karmiloff-Smith, A., Campos, R., Elsabbagh, M., Driver, J., \& Cornish, K. (2005). To look or not to look? Typical and atypical development of oculomotor control. Journal of Cognitive Neuroscience, 17, 591-604.

Schultz, R. (2005). Developmental deficits in social perception in autism: The role of the amygdala and fusiform face area. International Journal of Developmental Neuroscience, 23, 125-141.

Shah, A., \& Frith, U. (1993). An islet of ability in autistic children: A research note. Journal of Child PsYchology and Psychiatry and Allied Disciplines, 24, 613-620.

Spencer, J., O’Brien, J., Riggs, K., Braddick, O., Atkinson, J., \& Wattam-Bell, J. (2000). Motion processing in autism: Evidence for a dorsal stream deficiency. Neuroreport, 11, 2765-2767.

Spinelli, D., De Luca, M., Judica, A., \& Zoccolotti, P. (2002). Crowding effects on word identification in developmental dyslexia. Cortex, 38, 179-200.

Stein, J. (2001). The sensory basis of reading problems. Developmental Neuropsychology, 20, 509-534.

Stein, J., \& Walsh, V. (1997). To see but not to read: The magnocellular theory of dyslexia. Trends Neuroscience, 20, 147-152.

Takarae, Y., Minshew, N., Luna, B., \& Sweeney, J. A. (2004). Oculomotor abnormalities parallel cerebellar histopathology in autism. Journal of Neurology, Neurosurgery, Q Psychiatry, 75, 1359-1361.

Talcott, J. B., Gram, A., Van Ingelghem, M., Witton, C., Stein, J. F., \& Toennessen, F. E. (2003). Impaired sensitivity to dynamic stimuli in poor readers of a regular orthography. Brain and Language, 87, 259-266.

Valdois, S., Bosse, M. L., \& Tainturier, M. J. (2004). The cognitive deficits responsible for developmental dyslexia: Review of evidence for a selective visual attentional disorder. Dyslexia, 10, 339-363.

van der Geest, J. N., Kemner, C., Camfferman, G., Verbaten, M. N., \& van Engeland, H. (2001). Eye movements, visual attention, and autism: A saccadic reaction time study using the gap and overlap paradigm. Biological Psychiatry, 50, 614-619. 
Velay, J.-L., Daffaure, V., Giraud, K., \& Habib, M. (2002). Interhemispheric sensorimotor integration in pointing movements: A study on dyslexic adults. Neuropsychologia, 40(7), 827-834.

Wang, R., Liu, L., \& Liu, J. (2015). A new approach to the diagnosis of deficits in processing faces: Potential application in autism research. Science China Life Sciences, http://dx.doi.org/10.1007/ s11427-012-4337-4

White, S., Frith, U., Milne, E., Rosen, S., Swettenham, J., \& Ramus, F. (2006). A double dissociation between sensorimotor impairments and reading disability: A comparison of autistic and dyslexic children. Cognitive Neuropsychology, 23(5), 748-761.

Wilkes, B. J., Carson, T. B., Patel, K. P., Lewis, M. H., \& White, K. D. (2015). Oculomotor performance in children with high-functioning autism spectrum disorders. Research in Developmental Disabilities, 38, 338-344. http://dx.doi.org/10.1016/j.ridd.2014.12.022

Witkin, H. A., Oltman, P. K., Raskin, E., \& Karp, S. A. (1971). A manual for the Embedded Figures Tests. Palo Alto, CA: Consulting Psychologists Press.

Correspondence regarding this article should be directed to Stéphanie Bellocchi, PhD, University PaulValéry Montpellier 3, Department of Psychology, Route de Mende, Montpellier, France 34199 Montpellier Cedex 5, France. E-mail: stephanie.bellocchi@univ-montp3.fr 